\title{
Bestimmung der Konzentration eines neuen Antiarrhythmicums, Flecainid, im Plasma durch Hochleistungsflüssigkeitschromatographie (HPLC): Probenvorbereitung durch Extraktionssäulen
}

\author{
Von J. U. Becker \\ Kettelhack Riker Pharma GmbH, Borken/Westfalen
}

(Eingegangen am 26. Oktober 1983/22. Februar 1984)

\begin{abstract}
Zusammenfassung: Es wird eine Methode zur schnellen Bestimmung von Flecainid (Tambocor ${ }^{\circledR}$ ) in Humanplasma beschrieben. Flecainid wird aus alkalisiertem Plasma an eine C-18-Extraktionssäule gebunden. Nach Waschen mit Methanol/ $\mathrm{H}_{2} \mathrm{O}$, Volumenfraktion 0,5 und Acetonitril in Wasser, Volumenfraktion 0,45, wird die Substanz mit Methanol extrahiert und durch HPLC bestimmt. Die Probenvorbereitung dauert etwa 15 Minuten. Mit dieser Methode lassen sich Flecainidkonzentrationen im Bereich 100-2000 $\mu \mathrm{g} / \mathrm{l} \mathrm{direkt} \mathrm{mit}$ einer Genauigkeit von 5-10\% in Abhängigkeit von der Konzentration bestimmen. Die Methode liefert gleiche Werte wie eine länger eingeführte, aber zeitraubende Flüssigkeitsextraktionsmethode.
\end{abstract}

Measurement of the plasma concentration of a new antiarrhythmic agent, flecainid, using high performance liquid chromatography (HPLC) and sample preparation with extraction columns

Summary: A method is described for the rapid determination of flecainid (Tambocor ${ }^{\circledR}$ ) in human plasma. Flecainid is removed from alkalized plasma by binding to a C-18 extraction column. After washing the column with methanol/water $(1+1$, by vol.) and acetonitrile/water $(45+55$, by vol.), the drug is extracted with methanol and determined by HPLC. Sample preparation takes about 15 minutes. With this method, flecainid can be determined directly in the concentration range $100-2000 \mu \mathrm{g} / \mathrm{l}$, with an accuracy of $5-10 \%$ depending on the concentration. The results agree well with those obtained by the longer established but lengthy liquid extraction method.

\section{Einfïhrung}

Flecainidacetat ist eine neue hochwirksame antiarrhythmischē Substanz gegen ventrikuläre und supraventrikuläre Tachyarrhythmien (1)..Da alle Antiarrhythmica eine relativ enge therapeutische Breite besitzen, ist ès in manchen Fällen, besonders bei $\mathrm{Pa}$ tienten mit Nieren- oder Leberschäden, notwendig, das Antiarrhythmicum im Plasma zu bestimmen, um die Patienten auf eine optimale Dosis einstellen zu können (2).

Bisher wurden drei Methoden zur Bestimmung von Flecainid im Plasma publiziert: Die Substanz wurde direkt im deproteinierten Plasma durch HPLC mit Fluoreszenzdetektion bestimmt (3). Flecainid wurde mit Hexan aus alkalisiertem Plasma extrahiert und durch HPLC mit spektralphotometrischer Detektion gemessen (4) oder es wurde im Extrakt ohne chromatographische Trennung direkt spektralfluormetrisch bestimmt (5).

Da die meisten Laboratorien aber nur Hochleistungsflüssigkeitschromatographiegeräte mit spektralphotometrischer Detektion besitzen, ist die direkte Bestimmung (3) im deproteinisierten Plasma nicht möglich. Die spektralfluormetrische Messung ohne chromatographische Auftrennung ist störanfällig (5) und gelingt nur mit Gitter-Spektralfluorometern, bei denen die Emissionswellenlänge genau eingestellt werden kann. Bei Verwendung von Filterfluorometern verhindert eine zu hohe Untergrund- 
fluoreszenz die Bestimmung. Für die Chromatographie mit photometrischer Detektion muß die Probe konzentriert werden. Hierzu eignet sich die FlüssigExtraktionsmethode (4), die leider sehr zeitaufwendig ist und viel Úbung verlangt.

Es lag deshalb nahe, die Probenvorbereitung durch den Einsatz von Extraktionssäulen zu vereinfachen. Hier wird eine Methode zur Extraktion von Flecainid aus Plasma beschrieben, die den Zeitaufwand für die Probenvorbereitung gegenüber der Flüssigextraktion um einen Faktor von 15 reduziert und gleiche Werte liefert.

\section{Material und Methoden}

Flecainidacetat (N-(2-Piperidylmethyl)-2,5-bis(2,2,2 trifluorethoxy)-benzamidacetat (Molekulargewicht 474,4 ) und der interne Standard (N-(2-Piperidylmethyl)-2,5-diethoxybenzamid-hydrochlorid) wurden von Riker, St. Paul, USA, hergestellt. Humanplasma wurde vom lokalen Blutspendedienst, alle Lösungsmittel für die HPLC und Pentan-1-sulfonsäure wurden von Promochem, Wesel, bezogen.

Zur Plasmaextraktion wurde das Baker-10-Extraktionssystem mit C-18-Säulen von Baker (Katalog Nr. 7020-1, Baker Chemikalien, Groß Gerau) oder C-18-Bond-Elut-Säulen von Analytichem International (Katalog Nr. 607101, ICT Handelsgesellschaft, Frankfurt) benutzt.

\section{Extraktionssäulen}

Die Eignung einer Charge von Extraktionssäulen wurde folgendermaßen bestimmt: Mit einem Muster von 5 Säulen wurde der Flecainidgehalt von Plasma, das mit Flecainid $(1 \mathrm{mg} / \mathrm{l})$ und internem Standard versetzt war, nach der Vorschrift bestimmt. Der Flächenquotient der Peaks von Flecainid und dem internen Standard wurde berechnet und mit dem Flächenquotienten des Chromatogramms einer Mischung von gleichen Volumina der Stammlösungen des internen Standards $(5 \mathrm{mg} / \mathrm{l})$ und Flecainidacetat (10 $\mathrm{mg} /$ ) verglichen. Der Flächenquotient von höchstens einem der Säuleneluate durfte nur $80 \%$ des Flächenquotienten der Mischung betragen. In der Regel lag der Flächenquotient der aufbereiteten Proben bei $90-95 \%$ der Mischung. Die relative Standardabweichung überstieg $10 \%$ nicht.

\section{Lösungen}

\section{Methanol}

50,0 ml Methanol zur HPLC wurden mit $\mathrm{H}_{2} \mathrm{O}$ auf $100,0 \mathrm{ml}$ verdünnt.

\section{Acetonitril}

$45,0 \mathrm{ml}$ Acetonitril zur HPLC wurden mit $\mathrm{H}_{2} \mathrm{O}$ auf $100,0 \mathrm{ml}$ verdünnt.

\section{Carbonatlösung}

$\mathrm{Na}_{2} \mathrm{CO}_{3}(0,10 \mathrm{~mol} / \mathrm{l})$ wurden in $0,050 \mathrm{~mol} / \mathrm{INOH}$ gelöst.

\section{Standardlösungen}

Es wurde eine Stammlösung von Flecainidacetat $(1,00 \mathrm{~g} / \mathrm{l})$ in $\mathrm{H}_{2} \mathrm{O}$ hergestellt. Die Absorbanz dieser Lösung betrug nach 1:10 Verdünnung mit Wasser bei $298 \mathrm{~nm} 0,595 \pm 0,005$. Die Stammlö- sung wurde 1:100 mit Wasser verdünnt (Gehalt $10 \mathrm{mg} / \mathrm{l}$ ) und diente zur Kalibrierung. Die Absorbanz der Stammlösung des internen Standards $(250 \mathrm{mg} / \mathrm{l})$ wurde bei $313 \mathrm{~nm}$ in 1:2,5 Verdünnung überprüft. Sie betrug $0,983 \pm 0,011$ bei zwei verschiedenen Chargen der Substanz. Die Stammlösung wurde auf eine Konzentration von $5 \mu \mathrm{g} / \mathrm{l}$ verdünnt. Diese Verdünnung wurde als interner Standard den Proben und den Kalibrierungslösungen zugegeben. Beide Lösungen konnten im Kühlschrank mindestens ein halbes Jahr lang aufbewahrt werden.

\section{Chromatographie}

Flecainid wurde, wie in 1.c. (4) beschrieben, auf einer Zorbax TMS-Säule, 4,6 × $150 \mathrm{~mm}$, (DuPont), bestimmt. Als Vorsäule diente eine $2 \mathrm{~cm}$ Vorsäulenkartusche mit Lichrosorb RP 2, $10 \mu$, (Bischoff, Leonberg). Die mobile Phase bestand aus $450 \mathrm{ml} \mathrm{Ace-}$ tonitril, $5,5 \mathrm{ml}$ Eisessig und $544,5 \mathrm{ml}$ einer 0,1 molaren wäßrigen Lösung von Pentan-1-sulfonsäure. (Diese Mischung ist nur wenige Tage stabil und sollte deshalb im Kühlschrank gelagert werden.) Chromatographiert wurde mit einer Flußrate von $2 \mathrm{ml} / \mathrm{min}$ auf einem Spectra Physics SP 8100 Chromatographen mit Autosampler. Detektion erfolgte mit einem SP 8400 UV/vis Detektor bei $308 \mathrm{~nm}$ bei höchster Empfindlichkeitsstufe. Die Peakflächen wurden automatisch mit einem SP 4100 Integrator berechnet.

\section{Vorbereitung des Plasmas}

Die maximale Flecainidkonzentration im Plasma wird allgemein 2-4 Stunden nach der letzten Tabletteneinnahme erreicht. Der therapeutische Bereich liegt bei 300-1000 $\mu \mathrm{g} / \mathrm{I}$ Flecainidacetat (7).

Blut, $10 \mathrm{ml}$, wurde entnommen und die Gerinnung durch Zusatz von Heparin (etwa 150 USP-Einheiten) gehemmt. Nach Zentrifugation in einer Laborzentrifuge konnte das Plasma zur Bestimmung bis zu drei Tage bei Raumtemperatur aufbewahrt werden. Einfrieren oder Kühlung ist nur für längere Lagerung notwendig.

Unmittelbar vor der Bestimmung wurde $1,0 \mathrm{ml}$ Plasmaprobe in einem Reagenzglas mit $0,10 \mathrm{ml}$ Lösung des internen Standards, $0,5 \mathrm{ml}$ Wasser und 2,0 $\mathrm{ml}$ Carbonạtlösung gemischt.

\section{Extraktion des Flecainids}

Eine C-18-Extraktionssäule wurde mit 2 Füllungen Methanol (etwa $2 \times 1,5 \mathrm{ml}$ ) und einer Füllung Wasser gewaschen. Die Säule durfte nicht trocken laufen. Das alkalisierte Plasma wurde bei geringem Vakuum (10-20 kPa) durch die Säule gesaugt. AnschlieBend spülten wir die Säule mit einer Füllung Carbonatlösung und einer Füllung Wasser, zwei mal mit etwa $1 \mathrm{ml}$ Methanol/ $/ \mathrm{H}_{2} \mathrm{O}$ und zwei mal mit genau $1,0 \mathrm{ml}$ Acetonnitril in $\mathrm{H}_{2} \mathrm{O}$. Nachdem das letzte Lösungsmittel durchgelaufen war, wurde das Vakuum abgestellt und ein $12 \times 75 \mathrm{~mm}$ Reagenzglas unter die Säule gesetzt.

Mit $0,5 \mathrm{ml}$ reinem Methanol wurden sehr langsam (innerhalb von 1-2 Minuten) das Flecainid und der interne Standard eluiert. Das Methanol wurde bei $60^{\circ} \mathrm{C}$ unter einem Stickstoffstrom abgetrieben, der Rückstand in $0,25 \mathrm{ml}$ mobiler Phase gelöst und $0,1 \mathrm{ml}$ chromatographiert.

Alle Arbeitsgänge wurden in 12-15 Minuten erledigt, und es konnten bis zu 10 Proben parallel bearbeitet werden.

\section{Ergebnisse}

.Durch das beschriebene Verfahren wurde das Flecainid zur Chromatographie konzentriert und gleichzeitig wurden Verunreinigungen entfernt, die in $\mathrm{Hu}$ - 


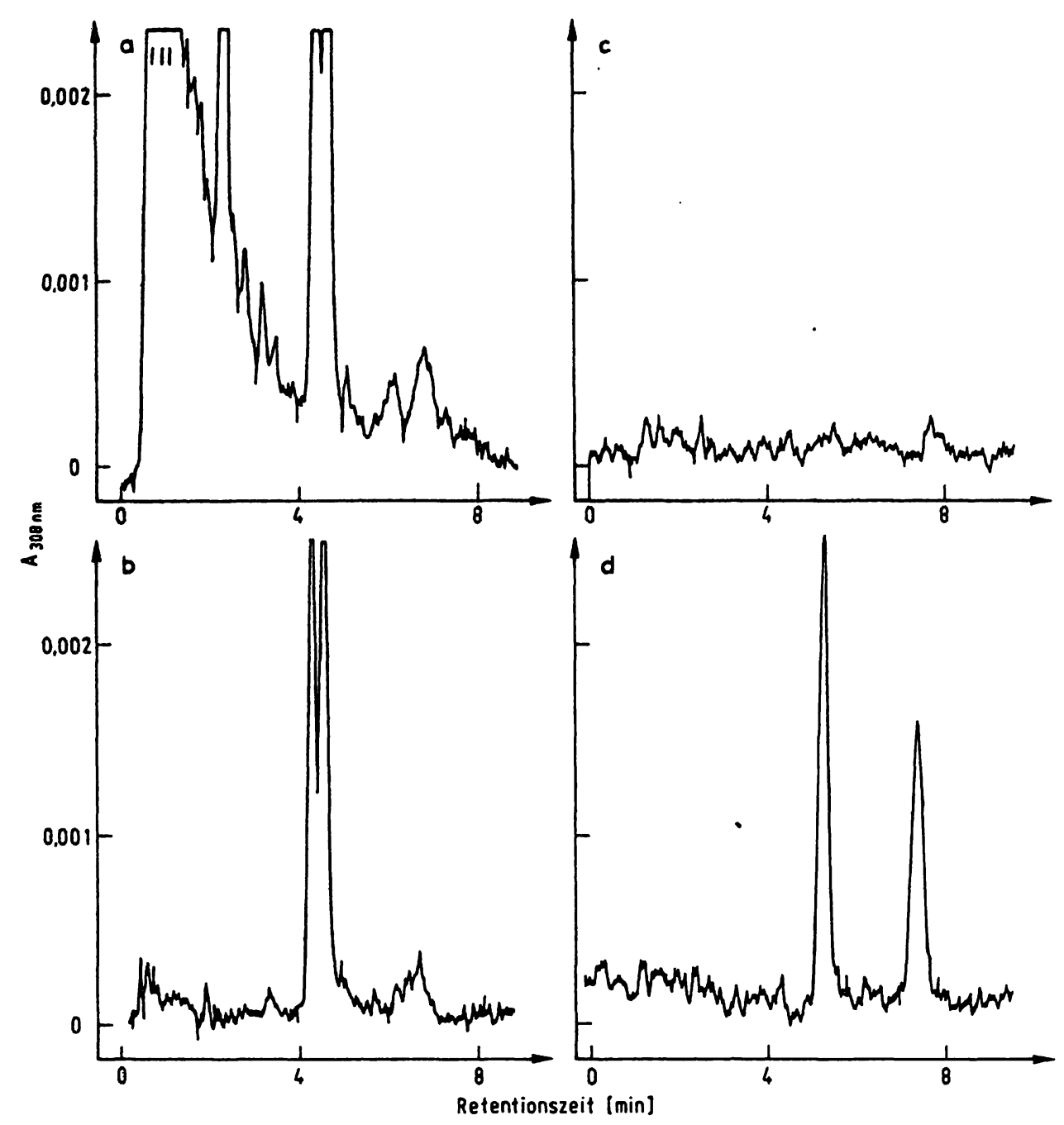

Abb. 1a-c. Entfernung störender Substanzen von der Extraktionssäule durch die verschiedenen Waschschrittc.

Humanplasma ohne Zusatz von internem Standard oder Flecainid wurde auf Extraktionssäulen aufgetragen. Die Säulc wurde nur mit Wasser (a), mit Wasser und wäßr. Methanol (Volumenfraktion 0,5) (b), mit Wasser, wäßr. Methanol und Acetonitril in Wasser, Volumenfraktion 0,45 ) (c) gewaschen. In jedem Experiment wurden die Säulen mit $0,5 \mathrm{ml} \mathrm{Methanol}$ eluiert. Das Eluat wurde unter Standardbedingungen chromatographiert. In der Abbildung werden Kopien der Originalchromatogramme dargestellt.

Abb. 1d. Typisches Chromatogramm des Eluates von Plasma mit $600 \mu \mathrm{g} /$ / Flecainidacetat.

manplasma die Flecainidbestimmung störten (Abb. $1 \mathrm{a}-\mathrm{c}$ ). Methanol $/ \mathrm{H}_{2} \mathrm{O}$ (Volumenfraktion 0,5 ) wusch die meisten störenden Substanzen aus (Abb. 1a), wobei allerdings Stoffe zurückblieben, die in Positionen des internen Standards chromatographierten (Abb. 1b). Diese Substanzen wurden durch Acetonitril in $\mathrm{H}_{2} \mathrm{O}$ (Volumenfraktion 0,45) entfernt, (Abb. 1c), wodurch wir nach diesem Waschvorgang ein sehr sauberes Chromatogramm erhielten, in dem nur noch die Peaks des internen Standards und des Flecainids sichtbar waren (Abb. 1d).

Die Ausbeuten von Flecainid wurden innerhalb der Fehlergrenze nicht beeinflußt, wenn die Mengen von $\mathrm{H}_{2} \mathrm{O}$, Carbonatlösung und wäßr. Methanol für die Waschschritte von $1-6 \mathrm{ml}$ variiert wurden. Die Menge von Acetonitril mußte jedoch genau bemessen sein, da Flecainid durch diesen Waschgang bei bestimmten Chargen von Extraktionssäulen teilweise ausgewaschen wurde. Aus diesem Grunde wurden Muster der Extraktionssäulen einer neuen Charge immer auf ihre Eignung für die Bestimmung geprüft, wie es bei den Methoden beschrieben ist. Die Extraktionssäulen einer Charge verhielten sich alle sehr ähnlich.

\section{Kalibrierung}

Die Flecainidkonzentration in den Proben wurde an Hand einer Standardkurve ermittelt. Eine solche Standardkurve für Flecainid im Bereich von 100$2000 \mu \mathrm{g} / 1$ Plasma wird in Abbildung 2 gezeigt. Dem Plasma wurde in diesem Falle statt des Wassers $0,5 \mathrm{ml}$ einer Lösung mit verschiedenen Mengen Flecainidacetat zugesetzt und die Proben extrahiert, wie 


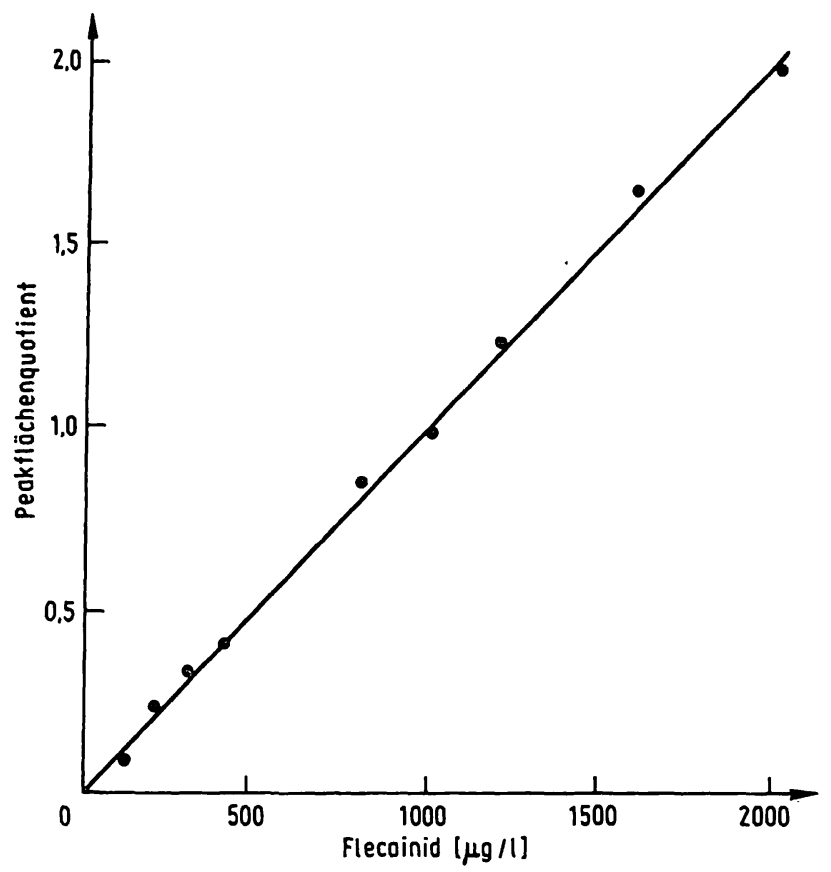

Abb. 2. Standardkurve für Flecainidacetat.

Humanplàsma wurde mit Flecainidacetat (100-2000 $\mu \mathrm{g} / \mathrm{l})$ versetzt und die Proben mit der hier beschriebenen Methode bearbeitet. Der Flächenquotient des Flecainidpeaks und des Peaks des internen Standard wurde berechnet und gegen die Flecainidacetatkonzentration aufgetragen. (100 $\mu \mathrm{g} / \mathrm{l}$ Flecainidacetat entsprechen 211 $\mathrm{nmol} / \mathrm{l})$.

oben beschrieben. Der Flächenquotient aus dem Flecainidpeak und dem Peak des internen Standards wurde gegen die Konzentration des Flecainidacetat im Plasma aufgetragen. Im Bereich von 100-2000 $\mu \mathrm{g} /$ l Plasma hängt der Flächenquotient linear von der Flecainidacetatkonzentration ab.

\section{Ausbeute, Genauigkeit und Richtigkeit der Methode}

Aus Humanplasma, das mit 200,600 und $1000 \mu \mathrm{g} / 1$ Flecainidacetat versetzt wurde, extrahierten wir bei jeder Konzentration zwischen 76 und $83 \%$ des Flecainids und $78-81 \%$ des internen Standards. Die Genauigkeit der Methode wurde an jeweils 7 Flecainid enthaltenden Proben mit 200, 600 und $1000 \mu \mathrm{g} / 1$ an einem Tag ermittelt. Die Variationskoeffizienten betrugen $10 \%, 6 \%$ und $5 \%$. Um die Streuung von Tag zu Tag zu bestimmen, wurden die Variationskoeffizienten der Peakflächenquotienten für 200, 600 und $1000 \mu \mathrm{g} / \mathrm{l}$ Flecainidacetat aus den Standardkurven über einen Monat $(n=15)$ berechnet. Sie betrugen $19 \%, 13 \%$ und $13 \%$. Die Streuung ist bei diesen Werten größer als die Streuung von Tag zu Tag, da verschiedene Chargen von Extraktionssäulen verwendet wurden und der Peakflächenquotient von der Zusammensetzung und dem Alter der mobilen Phase abhängt. Aus diesem Grunde muß täglich eine neue Standardkurve erstellt werden.
Ähnliche Genauigkeiten wurden bei Routineanalysen mit der Flüssigextraktionsmethode (4) erreicht. Die Richtigkeit hängt von der Standardsubstanz ab. Kontrolle der UV-Absorbanz der Stammlösungen, wie in Material und Methoden beschrieben, half die Richtigkeit zu verbessern.

\section{Interferenz durch andere Arzneistoffe}

Chang und Mitarbeiter (4) untersuchen den Einfluß anderer Arzneistoffe, die die Bestimmung stören können, da sie bei der Chromatographie nahe bei dem Peak des Flecainid oder des internen Standards eluieren. Hierzu gehören Diazepam, Dipryidamol, Propranolol, Chinin und Sulfinpyrazon. Diese Substanzen wurden in den gleichen Konzentrationen wie bei Chang et al. (4) Humanplasma zugesetzt. Außerdem wurde Amiodaron (2500 $\mu / /$ Plasma) im gleichen System getestet. Das Plasma wurde mit der neuen Methode aufgearbeitet und das Säuleneluat chromatographiert. Außer Chinin, das nur eine geringfügig längere Retentionsżeit als Flecainid besitzt, wurden alle anderen Stoffe gut von Flecainid und dem internen Standard abgetrennt und störten deshalb die Bestimmung nicht.

Vergleich zwischen der Probenvorbereitung durch Extraktionssäulen und Flüssig-Extraktion

Chang und Mitarbeiter (4) zeigten, daß die neue Flüssig-Extraktionsmethode mit anschließender Hochleistungschromatographie gleiche Werte liefert wie die gaschromatographische Methode, die während der Entwicklung und klinischen Erprobung von Flecainid benutzt wurde (6). Um festzustellen, ob man mit der beschriebenen Methode gleiche Werte erhält wie bei der Flüssig-Extraktionsmethode, wurden sie beide verglichen. Flecainid-Plasmaproben von 29 Patienten wurden parallel mit der FlüssigExtraktionsmethode (4) und der neuen Methode untersucht.

Aus dem Korrelationsdiagramm (Abb. 3) berechneten wir eine Gerade mit der Gleichung: $y=0,882 x$ $+87(\mu \mathrm{g} / \mathrm{l})$, deren Steigung und Achsenabschnitt sich geringfügig, aber signifikant von 1 bzw. 0 unterscheiden (erklärte Varianz: 1. Hauptkomponente $99,3 \%$, 2. Hauptkomponente $0,67 \%$; $x=$ Konzentration bestimmt durch Flüssigextraktion; $y=$ Konzentration bestimmt durch Extraktionsșäulen). Bei geringen Flecainidkonzentrationen ergaben sich etwas höhere, bei Konzentrationen um $700 \mu \mathrm{g} / \mathrm{l}$ etwa gleiche und bei höheren Konzentrationen etwas niedrigere Werte als mit der Flüssig-Extraktion. Die -Ursache für diese Abweichung ist unbekannnt. Die Unterschiede sind jedoch so gering, daß sie praktisch keine Bedeutung besitzen. 


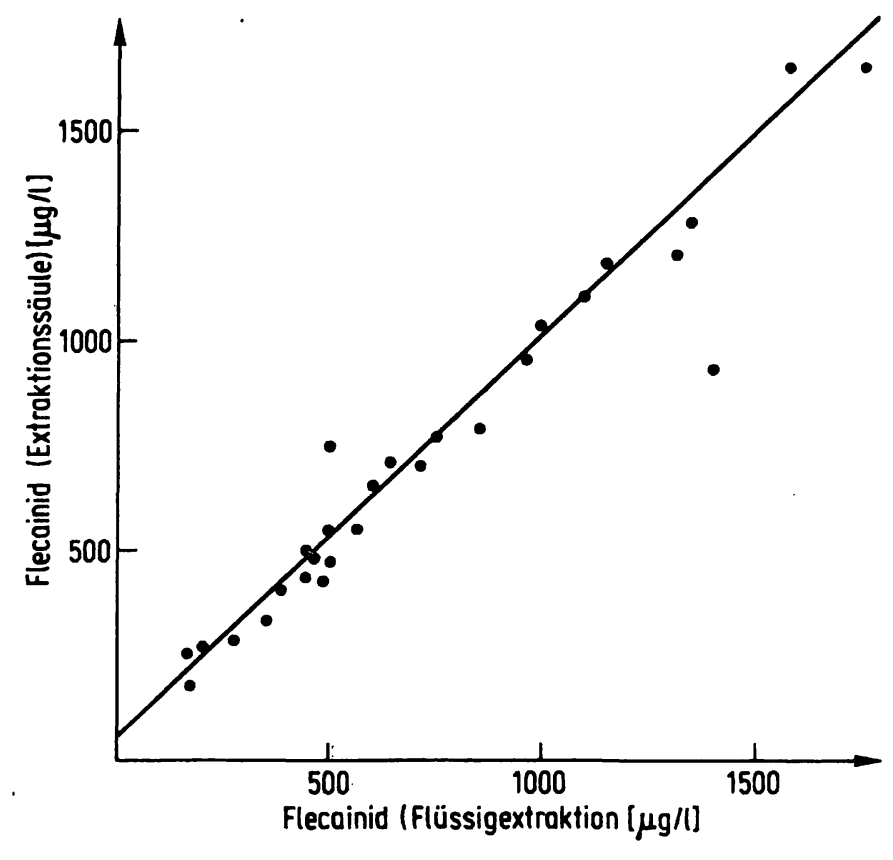

Abb. 3. Vergleich der Säulenextraktionsmethode mit der Flüssig-Extraktionsmethode (4).

Flecainid im Plasma von 29 Patienten aus verschiedenen Kliniken wurde sowohl mit der Säulenextraktionsmethode, als auch mit der Flüssig-Extraktionsmethode (4) untersucht und die Werte gegeneinander aufgetragen. (Ein weiterer Punkt der Geraden liegt bei $3400,3100 \mu \mathrm{g} / \mathrm{l})$.

\section{Diskussion}

Der Hauptvorteil der hier beschriebenen Methode zur Probenvorbereitung für die Bestimmung der Flecainidkonzentration in Humanplasma liegt in der großen Zeitersparnis. Während man bei der Flüssigextraktion mindestens 135 Minuten benötigt, sind die Proben beim Einsatẑ von Extraktionssäulen schon nach 15 Minuten zur Chromatographie bereit. Außerdem ist die Ausbeute am Flecainid größer, da man bei der Flüssigextraktion immer einen Teil der Probe beim Abhebern der Phasen verliert. Die Methode läßt sich sehr leicht erlernen, so daß auch unerfahrene Laboranten schnell gute Analysen liefern.
Die Genauigkeit der Bestimmung hängt im wesentlichen von der Güte des Detektors und des Chromatographiesystems ab. Besondere Sorgfalt muß allerdings auf die Auswahl der Extraktionssäulen gelegt werden, da wir Chargen geliefert erhielten, bei denen das Flecainid bei der Waschung mit mehr als $2 \mathrm{ml}$ wäßr. Acetonitril teilweise eluiert wurde. Aus diesem Grunde muß jede Charge auf Eignung für die Flecainidbestimmung untersucht werden und das Volumen des wäßr. Acetonitril muß genau abgemessen werden, um Fehlanalysen zu vermeiden. Alle Versuche, die Extraktionssäulen durch Waschen mit Lösungsmitteln zu regenerieren, sind bisher gescheitert. Wir benutzen daher immer neue Säulen für die Bestimmung. Die Genauigkeit der Bestimmung kann gesteigert werden, wenn man fluormetrische Detektion benutzt (Erregungswellenlänge $298 \mathrm{~nm}$, Emissionswellenlänge $370 \mathrm{~nm}$ ), wodurch die Empfindlichkeit etwa 100 fach erhöht und dadurch das Grundrauschen verkleinert wird. Die Bestimmungsmethode wird durch andere Arzneimittel wenig gestört. Lediglich sehr hohe Konzentrationen von Chinin oder Chinidin (einem Stereoisomeren des Chinin, das gleiche chromatographische Eigenschaften besitzt) könnten die Bestimmung erschweren. Allerdings wurde bisher in mehr als 500 untersuchten Plasmaproben die Bestimmung von Flecainid durch die Komedikation nicht verhindert. Lediglich in Plasmaproben, die länger als ein halbes Jahr gefroren aufbewahrt wurden, sollte Flecainid nicht mit Extraktionssäulen bestimmt werden, da die Säulen dann häufig verstopfen. In diesen Fällen sollte man das Flecainid mit Hexan extrahieren (4). In allen anderen Fällen kann man jedoch Extraktionssäulen anwenden. Wir hoffen, daß die hier beschriebene Methode die Flecainidbestimmung so vereinfacht, daß es vielen Krankenhäusern mit HPLC-Geräten möglich ist, die Flecainidkonzentration im Plasma von Patienten in kritischen Fällen zu bestimmen. Auf diese Weise könnte die antiarrhythmische Therapie noch effektiver und sicherer gestaltet werden.

\section{Literatur}

1. Klempt, H. W., Nayebagha, A. \& Fabry, E. (1982) Z. Kardiol. $71,340-349$.

2. Follath, F., Ganzinger, U. \& Schuetz, E. (1983) Clin. Pharmacokinetics 8, 63-82.

3. DeJong, J. W., Hegge, J. A. J., Harmsen, E. \& DeTombe, P. Ph. (1982) J. Chromatogr. 229, 498-502.

4. Chang, S. F., Welscher, T. M., Miller, A. M. \& Ober, R. E. (1983) J. Chromatogr. 272, 341-350.

5. Chang, S. F., Miller, A. M., Jernberg, M. J., Ober, R. E. \& Conard, G. J. (1983) Arzneim. Forsch. 33, 251-253.

6. Johnson, J. D., Carlson, G. L., Fox, J. M., Miller, A. M., Chang, S. F. \& Conard, G. J. (1983) J. Pharm. Sci. eingereicht.

7. Conard, G. J., Cronheim, G. E. \& Klempt, H. W. (1982) Arzneim. Forsch. 32, 1.55-159.

Privatdozent

Dr. J. U. Becker

Kettelhack Riker Pharma GmbH

D-4280 Borken/Westfalen 